\title{
Self-Reported Autism Symptoms in Adults with Autism Spectrum Disorders
}

\author{
Somer L. Bishop and \\ Division of Developmental and Behavioral Pediatrics, Cincinnati Children's Hospital Medical \\ Center, MLC 4002, Cincinnati, OH 45229, USA \\ Marsha Mailick Seltzer \\ Waisman Center, University of Wisconsin-Madison, Madison, WI, USA
}

Somer L. Bishop: somer.bishop@cchmc.org

\begin{abstract}
Scores on the autism spectrum quotient (AQ) were examined in 65 adults with ASD. Maternal reports of symptoms were collected simultaneously using the autism diagnostic interview-revised (ADI-R) and the Vineland Screener. A slightly revised AQ administration procedure was used to accommodate adults with below average IQ. AQ scores were lower than in the original validation study, with only 11 adults (17\%) scoring above the proposed diagnostic cut-off and 24 (27\%) exceeding the screening cut-off. Adults with higher IQs endorsed more symptoms than those with below average intelligence, but even when analyses were restricted to the 39 adults with at least average IQ, only $44 \%$ met the screening cut-off. AQ scores were not significantly correlated with ADI-R or Vineland scores.
\end{abstract}

\section{Keywords}

Autism spectrum quotient; Adults; Assessment; Self-report

\section{Introduction}

Autism spectrum disorders (ASD) have been conceptualized as pervasive, lifelong disorders, and a number of longitudinal studies of ASD have supported the stability of these diagnoses over time (Billstedt et al. 2005; Lord et al. 2006). Many individuals with ASD also have a diagnosis of intellectual disability (ID), with estimates ranging from 50 to $75 \%$ of the ASD population (Bertrand et al. 2001; Fombonne 2005). Over the last several years, the field has made significant advancements in efforts to conduct valid, standardized assessments of children with a suspected diagnosis of ASD (Gotham et al. 2011). However, because ASD is normally diagnosed during childhood, less attention has been given to the development of assessment tools that are valid for use in adult populations.

Unlike assessment of other types of adult psychopathology, such as mood and anxiety disorders, where standardized self-report measures are central to diagnostic practices, selfreport has not traditionally been part of assessment for ASD. Thus, until recently, there were no standardized self-report measures available for use in adults with ASD. To address this need, Baron-Cohen and colleagues developed the Autism-Spectrum Quotient (AQ; Baron-

(C) Springer Science+Business Media, LLC 2012

Correspondence to: Somer L. Bishop, somer . bi shop@ cchmc . org. 
Cohen et al. 2001), a brief, self-administered questionnaire designed to measure autistic traits in adults with normal intelligence.

The AQ applies a quantitative approach to the measurement of ASD symptoms. This assumes that behaviors associated with ASD lie on a continuum, where individuals with a diagnosis of ASD would be expected to be at one end, and unaffected individuals with few ASD symptoms or characteristics would be at the other end. Thus, the AQ is designed to provide a continuous measure of autistic traits that can be used to indicate where an individual with normal IQ falls on the ASD continuum (Baron-Cohen et al. 2001). Although the authors emphasize that high scores on the AQ do not necessarily mean that an individual warrants a diagnosis of ASD, they suggest that the AQ may be a useful tool for indicating the degree of "caseness" in research studies of ASD, or, in a clinical setting, for identifying adults who should undergo further evaluation.

Baron-Cohen et al. (2001) reported that in the initial AQ validation sample, $80 \%$ of individuals with an independent diagnosis of ASD $(n=58)$ scored above the proposed cutoff of 32 , whereas only $2 \%$ of controls selected randomly from the general population scored above that cut-off. A follow-up study by Woodbury-Smith et al. (2005) suggested that a lowered cut-off score of 26 was preferable when using the AQ for screening purposes. Among 100 patients with average intelligence evaluated at a diagnostic clinic, this revised cut-off yielded a sensitivity of 0.95 and a specificity of 0.52 (Woodbury-Smith et al. 2005).

The AQ has been revised for adolescents (Baron-Cohen et al. 2006) and for Japanese, Dutch, and French speaking populations (Ketelaars et al. 2008; Lepage et al. 2009; Wakabayashi et al. 2004). It has been employed in a number of recent studies of both clinical and general population samples to examine the relationships between ASD symptoms and a wide range of variables, including personality characteristics, selfreferential cognition, and empathy (Austin 2005; Lombardo et al. 2007; Wakabayashi et al. 2006). In addition, the AQ has been used as a measure of the broader autism phenotype in parents of individuals with ASD (Bishop et al. 2004; Wheelwright et al. 2010; Whitehouse et al. 2007). More recently, the AQ has even been used to measure the relationships between autistic traits and various neural and physiological correlates, such as gray matter volume, magnocellular visual evoked potential delay, and salivary testosterone (Sasamoto et al. 2011; Sutherland and Crewther 2010; Takagishi et al. 2010).

With regard to independent investigations of the psychometric properties of the AQ, many studies have relied on non-ASD general population and/or student samples (Hurst et al. 2007; Kloosterman et al. 2011). While these studies increase understanding of the factor structure, internal consistency, and reliability of the AQ, ASD samples are needed to draw conclusions about its validity as a screening tool, or as a measure of ASD symptoms. Studies that have examined the use of the AQ in individuals with ASD have raised some questions about its sensitivity. Ketelaars et al. (2008) reported that scores on the Dutch translation of the AQ did not differentiate between patients diagnosed with mild ASD and those without ASD, except on the Communication subscale. On the other hand, Hoekstra et al. (2008) found the Dutch AQ to have good sensitivity and specificity in differentiating adults with ASD from adults who were either in the general population or who had diagnoses of social anxiety disorder or obsessive-compulsive disorder. Using the Japanese translation of the AQ, Kurita et al. (2005) reported a sensitivity of 0.76 using a cut-off of 26 . However, AQ scores among their 26 participants with established ASD diagnoses were on average 6 points lower than participants with ASD in the British validation sample.

Taken together, results of these studies indicate a need for more research on samples of adults with ASD to evaluate the appropriateness of the AQ for use in clinical practice and 
research. Particularly if the AQ is going to be used as a screening instrument, it is important to determine whether recent findings about adults with ASD scoring lower than expected on the Dutch and Japanese translations of the AQ apply to the English version, as well.

The current study examined AQ scores in a community sample of adults with ASD. This sample provided a unique opportunity to investigate the criterion related validity of the AQ, because all of the adults entered the study with previous diagnoses of ASD, and because maternal reports of ASD symptoms were collected at the same time as adult self-reported symptoms on the AQ. Given that a large proportion of adults with ASD have a comorbid intellectual disability (e.g. Fombonne 2005), we also examined the use of the AQ in participants with below average intelligence. However, because the AQ was specifically designed for adults with normal intelligence, additional analyses were conducted separately for participants with below average versus average or above average IQ.

\section{Methods}

Participants were drawn from an ongoing longitudinal study of adolescents and adults with ASD living in Wisconsin or Massachusetts (Seltzer et al. 2003, 2011; Taylor and Seltzer 2011). Begun in 1998, the Adolescents and Adults with Autism (AAA) study has thus far collected information from the families of 405 adolescents and adults with ASD eight times over the course of 12 years. Upon entry into the study, eligibility criteria included that the family had at least one child with a community diagnosis of ASD (i.e., a previous diagnosis from a physician, psychologist, or other professional in the community), and that this diagnosis could be confirmed based on lifetime ratings on the Autism Diagnostic InterviewRevised (ADI-R; see below).

At the fifth wave of data collection, which took place between 2004 and 2005, the study protocol included a direct assessment of a sub-sample of adults with ASD. Selection criteria for this sub-sample included verbal ability and willingness to participate. Because the present set of analyses was focused on adults, only participants who met the above criteria and were at least 18 years of age at the time of the direct assessment were included. A total of 271 individuals were at least 18 years old at the fifth wave of data collection; 85 of these had sufficient verbal ability and interest to participate in the direct assessment. However, given our primary focus on the AQ, participants were only included in the analyses if complete AQ data were available. This resulted in a final sample of 65 adults with ASD between the ages of 18 and 52. As shown in Table 1, the majority of these individuals were male and Caucasian. Full-scale IQ (FSIQ) varied widely, ranging between 35 and 142, but participants were generally quite high functioning $(M=89.61)$. Only $22 \%$ of the sub-sample received scores in the range of intellectual disability (i.e., FSIQ $<70$ ).

Participants were interviewed in their homes by trained interviewers who had achieved appropriate standards of reliability on the measures included in the study protocol. Adults with ASD were administered the AQ, the Wide Range Intelligence Test (WRIT: Glutting et al. 2000), and an interview about friendships (Friendship Interview: see Losh and Piven 2007). Mothers of the adults with ASD completed a lengthy questionnaire, as well as an interview that included the Vineland Screener (Sparrow et al. 1993) and algorithm items from the Autism Diagnostic Interview-Revised (Rutter et al. 2003).

\section{Measures}

Autism-Spectrum Quotient-Development of the AQ (Baron-Cohen et al. 2001) was based on observations of individuals with ASD. The measure includes 50 items that were selected to be representative of the triad of impairments in ASD (i.e., deficits in communication and reciprocal social interaction, and the presence of restricted and 
repetitive behaviors), as well as associated cognitive styles and preferences that have been observed in individuals with ASD (e.g., attention to detail). The respondent is asked to indicate whether he/she agrees, strongly agrees, disagrees, or strongly disagrees with a series of statements. For scoring purposes, agree and strongly agree are combined, and disagree and strongly disagree are combined. Responses that indicate the presence of an ASD symptom are scored 1 point. The maximum possible score on the measure is 50 ( 1 point for each item).

The AQ includes five conceptually derived subscales: Social Skill; Attention Switching; Attention to Detail; Communication; and Imagination. Each subscale contains ten items. Baron-Cohen et al. (2001) reported moderate to high internal consistency for the subscales, ranging from 0.63 for Attention to Detail to 0.77 for Social Skill. Test-retest reliability was also good $(r=0.70)$, as was convergence between parent and child report. While parents tended to rate their adult children as having more difficulties than the adult children rated themselves, the average difference between self- and parent-report scores on the AQ was only 2.8 points (see Baron-Cohen et al. 2001).

Because the current study involved administering the AQ to a more intellectually heterogeneous group of individuals, it was necessary to make some modifications to standard AQ administration procedures to accommodate varying degrees of intellectual abilities among participants. To ensure that the adults in our sample would be able to comprehend the questions, we amended eight items that contained negatives so that they were phrased without use of the negative (scoring was also reversed for these 8 items). For example, "It does not upset me if my daily routine is disturbed" was changed to "It upsets me if my daily routine is disturbed." Table 2 contains a list of the 50 AQ items; wording differences adopted for the current study appear in italics. Additionally, interviewers read the questions to the participants as opposed to having the participants read the questions themselves. This was done to ensure that reading difficulties would not interfere with some participants' ability to complete the measure. Despite these alterations to the standard administration, internal consistency of the overall AQ was acceptable (Cronbach's Alpha = 0.74). Cronbach's alpha coefficients for the sub-scales tended to be lower (Social Skill, 0.76; Attention Switching, 0.57; Attention to Detail, 0.59; Communication, 0.61; Imagination, 0.42 ), and were generally lower than reported in the validation sample in which coefficients ranged from 0.63 to 0.77 . However, it is important to note that only the total score, and not the subscale scores, is used when determining whether or not an individual meets screening and/or diagnostic cut-offs on the AQ.

The Wide Range Intelligence Test-The WRIT (Glutting et al. 2000) is a measure of intelligence that provides standard scores of verbal, nonverbal, and general intelligence. This measure has been validated for use with individuals of different ages, ethnicities, and educational backgrounds (Shields et al. 2004). In the present study, we used the general intelligence standard score, with a mean of 100 and a standard deviation of 15 , as a measure of overall cognitive ability.

Vineland Screener-The Vineland Screener (Sparrow et al. 1993) is an abbreviated form of the Vineland Adaptive Behavior Scales (Sparrow et al. 1984). It is a semi-structured interview that is administered to parents or caregivers to obtain an estimate of an individual's current adaptive abilities. The Vineland Screener yields scores in Communication, Daily Living Skills, and Socialization, as well as an overall Adaptive Behavior Composite score. Higher scores indicate better adaptive abilities.

Autism Diagnostic Interview-Revised-The Autism Diagnostic Interview-Revised (ADI-R: Rutter et al. 2003) is a comprehensive semi-structured interview for caregivers of 
individuals with suspected ASD. The interview is organized into three symptom domains that reflect DSM-IV criteria for autism (i.e., Qualitative Abnormalities in Reciprocal Social Interaction, Qualitative Abnormalities in Communication, and Restricted, Repetitive, and Stereotyped Patterns of Behavior). The diagnostic algorithm, which is based on lifetime ratings of behaviors, has been shown to have high levels of sensitivity and specificity (Lord et al. 1994).

At the initial wave of data collection, mothers of adolescents and adults with ASD were asked both about their child's current and past behaviors. Information about past behaviors was collected in order to verify that the adolescent or adult met ADI-R diagnostic algorithm cut-offs for ASD (see Seltzer et al. 2003). At subsequent waves, caregivers were asked only about their child's current behaviors.

For the present set of analyses, ADI-R current domain scores were calculated by adding current scores for behaviors in the diagnostic algorithm that are applicable to individuals 10 years and older (see "current algorithm for individuals 10 years and over" Rutter et al. 2003). We also calculated an overall index of ASD symptoms by summing the current scores from all three domains (using the Verbal total from the Communication domain). The "current algorithm" for the ADI-R has not been validated for diagnostic purposes, so there are no established cut-offs to determine an ADI-R classification of ASD or non-ASD, but current total scores can provide estimates of the individual's present level of ASD symptoms (as observed by the parent), where higher scores indicate more abnormality.

\section{Results}

Analyses were conducted in SPSS. Because of multiple comparisons, significance was set at $p \leq .01$.

\section{$A Q$ Total Scores}

Total scores in our sample were substantially lower $(\mathrm{M}=24.62, \mathrm{SD}=6.22)$, signifying fewer autism symptoms, than the mean of 35.8 that was reported for individuals with ASD in the original validation study. Of the 65 adults for whom complete AQ data were available, $11(17 \%)$ met the diagnostic cut-off of 32 proposed by Baron-Cohen et al. (2001) and 24 (37\%) met the screening cut-off of 26 proposed by Woodbury-Smith et al. (2005).

\section{$A Q$ Subscale Scores}

To examine the pattern of scores among adults in our sample, we calculated subscale scores using the scales proposed by Baron-Cohen et al. (2001). As was the case with AQ total scores, subscale scores were much lower among adults in our sample compared to adults in the validation sample. Whereas Baron-Cohen reported mean subscale scores in individuals with ASD ranging between 6.4 and 8.0, our adults obtained mean subscale scores that ranged between 3.63 (Imagination) and 6.65 (Attention to Detail) (see Fig. 1).

An examination of the pattern of subscale scores revealed that individuals tended to endorse more ASD symptoms and thus received significantly higher scores in the areas of Attention Switching and Attention to Detail compared to the areas of Social Skill, Communication, and Imagination (see Fig. 1). For example, in the area of Social Skill, only $26 \%$ of individuals scored in the "autism direction" by disagreeing with the statement "I prefer to do things with others rather than alone." On the Communication subscale, only $31 \%$ disagreed with "I enjoy social chit chat." In contrast, individuals were more likely to receive scores on items in the Attention Switching and Attention to Detail sub-domains: $72 \%$ agreed with the statement "I frequently get so strongly absorbed in one thing that I lose sight of other things," and 74\% agreed with "I tend to notice details that others do not." 


\section{Relationships between AQ Scores and Characteristics of the Adult with ASD}

AQ scores were not associated with the adult's gender, $F(1,63)=0.10, p=.75$, residential status (i.e., co-residing with a parent vs. living outside of the home), $F(1,63)=2.26, p=.14$, or age $(r=-0.001, p=.99)$. AQ scores were significantly positively correlated with IQ ( $r=$ $0.33, p=.01$ ) (see Table 3; Fig. 2), indicating that individuals with higher IQs tended to endorse more symptoms on the AQ. Only the Social Skill and Communication subscale scores were significantly correlated with IQ $(r=0.49, p<.001 ; r=0.34, p=.007$, respectively), whereas scores on the other three subscales were not.

\section{Relationships Between AQ Scores and Maternal Report Measures}

To further explore how well AQ scores matched with other reports of current ASD symptoms, we examined correlations between the AQ, the ADI-R, and the Vineland Screener. As seen in Table 3, the AQ was not significantly correlated with overall ASD symptoms on the ADI-R or social ability as measured by the Vineland Screener. As expected, ADI-R total scores were significantly correlated with Vineland social scores ( $r=$ $-0.68, p<.001)$, but neither of these was significantly correlated with scores on the AQ.

\section{Adults with Below Average Versus Average/Above Average Intelligence}

Because the AQ was developed for use in adults with average intelligence, and given the clear association between IQ and AQ scores in the present sample, we examined AQ scores separately for individuals who received a full-scale IQ score of at least $85(n=39)$ compared to those who scored in the borderline range or range of intellectual disability $(n=23)$ on the WRIT. Three individuals who did not complete the WRIT were not included in these analyses. Table 4 presents for each IQ group the mean AQ total and subscale scores, as well as the \% meeting diagnostic or screening cutoffs. As shown in Table 4, the AQ total score was three points higher (signifying more autism symptoms) for adults with average or above average IQ than those with ID, and a greater percentage met diagnostic and screening criteria. As was the case for the sample as a whole, AQ scores were not significantly correlated with ADI-R or Vineland scores in either the below average IQ group or the average/above average IQ group.

\section{Discussion}

The current study examined AQ scores in a sample of adults with ASD. Based on the fact that all participants had previously received independent community diagnoses of ASD, met lifetime ADI-R criteria for autism, and were currently reported to exhibit significant socialcommunication difficulties on the ADI-R and the Vineland Screener, AQ scores in this sample were surprisingly low. Only 37 and $17 \%$, respectively, exceeded the proposed screening or diagnostic cut-offs, and both total and subscale scores were significantly lower than reported in the British validation and follow-up studies. The lack of strong correlations between scores on the AQ and scores on the ADI-R and Vineland Screener is not entirely surprising given the different types of difficulties assessed by each instrument, as well as the fact that one is parent-report and the other self-report. However, the low sensitivity of the AQ cut-offs in a group of individuals known to have substantial ASD-related impairment is significant.

The mean AQ score of 24.62 in our sample is similar to Ketelaars et al. (2008) and Kurita et al. (2005), which reported mean AQ scores of 22.5 and 29.4 in their participants with ASD. These authors hypothesized that lower scores in their samples may have reflected less severe ASD symptoms compared to participants in the validation sample, but this is unlikely to explain the low AQ scores reported here. Mothers of participants in the current study endorsed both high levels of ASD symptoms and impaired adaptive behavior skills in their 
adult children at the same time that the AQ was completed. Furthermore, numerous reports from the AAA project that included participants described in the current study have highlighted the tremendous difficulties faced by these adults and their families, including persistent ASD symptoms across domains, limited social participation, few or no friendships, and exceptionally low levels of gainful employment (Orsmond et al. 2004; Taylor and Seltzer 2010).

While it is unlikely that lack of ASD symptoms accounted for lower AQ scores in our sample, other sample characteristics may have contributed to the discrepancies seen here. Importantly, unlike many studies of adults with ASD, our participants were drawn from the community. Therefore, in comparison to adults in clinic settings who are seeking diagnosis or treatment related to their disorder, adults in the current study may have been less focused on their difficulties and/or less willing to admit to certain types of difficulties. Another potential explanation for lower mean scores in this sample is that although the AQ is not intended for people with below average intelligence, we opted to include all adults for whom complete AQ data were available (about one-third of the sample had IQs below 85). Indeed, AQ scores in our sample were significantly positively correlated with IQ, indicating that adults with higher IQs tended to endorse more symptoms on the AQ. Yet, even when subsequent analyses were limited to the 39 adults with IQs of 85 or above, AQ scores were still much lower than would be expected for adults with a diagnosis of ASD. Only 44\% of the adults with average or above average intelligence met screening cut-offs and $21 \%$ met diagnostic cut-offs, yielding much lower sensitivity than was reported in the validation studies. It is also interesting that proband IQ was not significantly related to maternal reports of difficulties on either the ADI-R or the Vineland. Thus, despite being rated similarly on other validated measures of ASD symptoms/social-communication difficulties, adults with higher IQs and lower IQs differed in their self-ratings.

Another important consideration is that limited insight, which is often associated with a diagnosis of ASD, may interfere with the ability of some adults with ASD to accurately report about their difficulties. In the current study, scores on the Communication and Social Skill sub-scales were significantly lower than scores on the Attention Switching and Attention to Detail subscales, indicating that adults with ASD may have a particularly difficult time recognizing their social and communication challenges. Baron-Cohen et al. (2001) also acknowledged this possibility that limited insight, or "mind-reading problems," in individuals with ASD could impair their judgments about their own behavior, so the AQ is specifically designed to include some items that inquire about preferences, such as "I enjoy social occasions" or "I'd rather go to a library than to a party." The inclusion of these items was intended to increase the sensitivity of the measure by not relying solely on a person's ability to accurately report about their behaviors. Interestingly, however, these items were among the least frequently endorsed in the "autism direction" by participants in the current study. For example, whereas the majority of participants acknowledged that it was hard for them to make new friends, only $17 \%$ indicated that they did not enjoy meeting new people. Clearly, with regard to items that assess preferences, there appears to be a great deal of variability in this population. It cannot be assumed that an adult with ASD will necessarily exhibit low levels of social interest or motivation.

Findings from the current study suggest that the AQ may not always provide a valid measure of ASD-related impairment. Whereas some adults with ASD may respond in the "nonautism" direction because they have poor insight into their difficulties, others may have higher levels of social interest than the measure assumes. Particularly when administered to adults with limited insight and higher levels of social interest, AQ scores may not accurately reflect the likelihood that an individual has ASD. Researchers and clinicians should exercise caution in using the AQ in isolation to screen for the presence of ASD or ASD related 
difficulties. It is also important to reiterate the fact that the AQ was specifically designed for adults with at least average cognitive abilities, and our findings further suggest that the measure is more sensitive in adults who meet this criterion.

The low rates of AQ item endorsement in our study also call into question whether the AQ should be used to measure autistic traits in clinical or general population samples. This is an important issue, because in studies that employ the AQ as a continuous measure of autism symptoms or as an index of ASD susceptibility, there could be a risk of drawing erroneous conclusions about the relationships between ASD-related constructs and other variables. In other words, if many individuals with ASD fail to respond to AQ items in the expected "autism direction," then it is necessary to evaluate whether higher scores are truly indicative of more ASD symptoms, or whether they might instead represent other factors (e.g., lack of interest in social interactions) that are not necessarily associated with ASD in adulthood.

\section{Limitations}

A significant limitation of the current study is that we altered the standard administration procedures of the AQ. Questions phrased in the negative direction were re-worded to improve comprehension, and participants were read the AQ questions (rather than reading them on their own). This was done because we extended the use of the AQ beyond the population for which it was originally intended and wanted to modify the administration to be more appropriate for individuals with ID. As a result of being read the questions, some individuals may have felt uncomfortable endorsing certain symptoms and thus may have received lower scores than they would have if standard administration procedures had been carried out. On the other hand, if social desirability were driving a tendency to under-report symptoms, we would have expected adults with higher IQs (and presumably more aware of social expectations) to report fewer symptoms than those with lower IQs, rather than more symptoms. Furthermore, it is unlikely that the revised administration procedure affected participants to such a degree that it substantially accounts for their lower AQ scores. It is also important to note that internal consistency of the total measure remained high despite these alterations. Nevertheless, before more solid conclusions can be drawn about the strengths and limitations of the AQ as a measure of ASD symptoms in adults, it will be important to conduct other studies of adults with ASD using the standard AQ administration procedure. It would also be worthwhile to explore the extent to which our altered administration actually improved comprehension. If these minor wording modifications improve comprehension in general, or in individuals with lower cognitive abilities, future studies may wish to employ similar modifications when including participants with intellectual disabilities.

The inclusion of adults who have both ASD and intellectual disabilities in future research is needed, given the high prevalence of ID in the ASD population. When designing self-report instruments for individuals with ASD who have ID, it will be important to draw on literature about interviewing individuals with compromised intellectual functioning. For example, research by Carol Sigelman and colleagues (e.g., Heal and Sigelman 1995) has shown that individuals with intellectual disabilities are particularly susceptible to response biases (e.g., acquiescence). Instruments that incorporate multiple-choice formats (especially with accompanying pictures) and that use item-reversal, yield more valid responses in individuals with ID than open ended or yes-no question formats. Embedding measures of consistency, such as including multiple questions with similar content, is another way to ensure that response biases have not threatened the reliability of the data. 


\section{Conclusion}

This investigation suggests multiple avenues for future research to discover strategies for obtaining valid self-reports of ASD symptoms and to probe more deeply into the subjective perceptions of adults with ASD. For some individuals, self-report provides a valid method for ascertaining information about ASD-related impairment, and may serve as the only means of gathering information about certain symptoms that are not necessarily visible to others (e.g., sensory preferences). For other individuals, such as those with limited insight into their difficulties, self-report may not be an appropriate way to establish or confirm an ASD diagnosis, or to index a person's level of ASD symptomatology. However, adults with ASD have a perspective that is important to consider in both assessment and treatment, and there is clearly a need to develop self-report measures that can be used in individuals with different levels of ability. Collecting information directly from diverse groups of adults with ASD, rather than making assumptions about the interests, motivations, or preferences of these individuals, will inform efforts to understand more about the various manifestations of and internal experiences associated with ASD in adulthood.

\section{Acknowledgments}

This work was supported by grants from the National Institute of Health, R01AG08768 and P30HD03352 to Marsha Mailick Seltzer, T32HD07489 to Len Abbeduto, and R01HD065277 to Somer L. Bishop. The authors would like to thank the families who participated in this research. We are grateful to the members of the Seltzer and Greenberg lab at the Waisman Center, particularly Jan Greenberg, as well as Amie Duncan at Cincinnati Children's Hospital, who provided input and support for this manuscript.

\section{References}

Austin EJ. Personality correlates of the broader autism phenotype as assessed by the autism spectrum quotient (AQ). Personality and Individual Differences. 2005; 38(2):451-460.10.1016/j.paid. 2004.04.022

Baron-Cohen S, Hoekstra RA, et al. The autism-spectrum quotient (AQ)*Adolescent version. Journal of Autism and Developmental Disorders. 2006; 36(3):343-350. [PubMed: 16552625]

Baron-Cohen S, Wheelwright S, Skinner R, Martin J, Clubley E. The Autism-Spectrum Quotient (AQ): Evidence from asperger syndrome/high-functioning autism, males and females, scientists and mathematicians. Journal of Autism and Developmental Disorders. 2001; 31(1):5-17.10.1023/A: 1005653411471 [PubMed: 11439754]

Bertrand J, Mars A, Boyle C, Bove F, Yeargin-Allsopp M, Decoufle P. Prevalence of autism in a United States population: The Brick Township, New Jersey, investigation. Pediatrics. 2001; 108(5): 1155.10.1542/peds.108.5.1155 [PubMed: 11694696]

Billstedt E, Gillberg C, Gillberg IC. Autism after adolescence: Population-based 13-to 22-year followup study of 120 individuals with autism diagnosed in childhood. Journal of Autism and Developmental Disorders. 2005; 35(3):351-360.10.1007/s10803-005-3302-5 [PubMed: 16119476]

Bishop DVM, Maybery M, Maley A, Wong D, Hill W, Hallmayer J. Using self report to identify the broad phenotype in parents of children with autistic spectrum disorders: A study using the autism spectrum quotient. Journal of Child Psychology and Psychiatry. 2004; 45(8):1431-1436.10.1111/j. 1469-7610.2004.00325.x [PubMed: 15482503]

Fombonne E. Epidemiology of autistic disorder and other pervasive developmental disorders. Journal of Clinical Psychiatry. 2005; 66:3-8. [PubMed: 16401144]

Glutting, J.; Adams, W.; Sheslow, D. Wide Range Intelligence Test (WRIT). Los Angeles, CA: Western Psychological Services; 2000.

Gotham, K.; Bishop, SL.; Lord, C. Diagnosis of autism spectrum disorders. In: Amaral, DG.; Geshwind, DH.; Dawson, G., editors. Autism spectrum disorders. New York: Oxford University Press; 2011. p. 30-43.

Heal LW, Sigelman CK. Response biases in interviews of individuals with limited mental ability. Journal of Intellectual Disability Research. 1995; 39(4):331-340. [PubMed: 7579991] 
Hoekstra RA, Bartels M, Cath DC, Boomsma DI. Factor structure, reliability and criterion validity of the Autism-Spectrum Quotient (AQ): A study in Dutch population and patient groups. Journal of Autism and Developmental Disorders. 2008; 38(8):1555-1566.10.1007/s10803-008-0538-X [PubMed: 18302013]

Hurst RM, Mitchell JT, Kimbrel NA, Kwapil TK, Nelson-Gray RO. Examination of the reliability and factor structure of the autism spectrum quotient (AQ) in a non-clinical sample. Personality and Individual Differences. 2007; 43(7):1938-1949.

Ketelaars C, Horwitz E, Sytema S, Bos J, Wiersma D, Minderaa R, et al. Brief report: Adults with mild autism spectrum disorders (ASD): Scores on the autism spectrum quotient (AQ) and comorbid psychopathology. Journal of Autism and Developmental Disorders. 2008; 38(1):176180. [PubMed: 17385086]

Kloosterman PH, Keefer KV, Kelley EA, Summerfeldt LJ, Parker JDA. Evaluation of the factor structure of the Autism-Spectrum Quotient. Personality and Individual Differences. 2011; 50(2): 310-314.10.1016/j.paid.2010.10.015

Kurita H, Koyama T, Osada H. Autism spectrum quotient-Japanese version and its short forms for screening normally intelligent persons with pervasive developmental disorders. Psychiatry and Clinical Neurosciences. 2005; 59(4):490-496. [PubMed: 16048456]

Lepage JF, Lortie M, Taschereau-Dumouchel V, Théoret H. Validation of French-Canadian versions of the Empathy Quotient and autism spectrum quotient. Canadian Journal of Behavioural Science/ Revue canadienne des sciences du comportement. 2009; 41(4):272-276.10.1037/a0016248

Lombardo MV, Barnes JL, Wheelwright SJ, Baron-Cohen S. Self-referential cognition and empathy in autism. PLoS ONE. 2007; 2(9):e883.10.1371/journal.pone.0000883 [PubMed: 17849012]

Lord C, Risi S, DiLavore PS, Shulman C, Thurm A, Pickles A. Autism from 2 to 9 years of age. Archives of General Psychiatry. 2006; 63(6):694-701.10.1001/archpsyc.63.6.694 [PubMed: 16754843]

Lord C, Rutter M, Couteur A. Autism diagnostic interview-revised: A revised version of a diagnostic interview for caregivers of individuals with possible pervasive developmental disorders. Journal of Autism and Developmental Disorders. 1994; 24(5):659-685. [PubMed: 7814313]

Losh M, Piven J. Social cognition and the broad autism phenotype: Identifying genetically meaningful phenotypes. Journal of Child Psychology and Psychiatry. 2007; 48(1):105-112. [PubMed: 17244276]

Orsmond GI, Krauss MW, Seltzer MM. Peer relationships and social and recreational activities among adolescents and adults with Autism. Journal of Autism and Developmental Disorders. 2004; 34(3): 245-256. [PubMed: 15264493]

Rutter, M.; Le Couteur, A.; Lord, C. Autism diagnostic interview-revised (ADI-R). Los Angeles, California: Western Psychological Services; 2003.

Sasamoto A, Miyata J, Hirao K, Fujiwara H, Kawada R, Fujimoto S, et al. Social impairment in schizophrenia revealed by Autism-Spectrum Quotient correlated with gray matter reduction. Social Neuroscience. 2011; 6(5-6):548-558.10.1080/17470919.2011.575693 [PubMed: 21943127]

Seltzer, MM.; Greenberg, JS.; Taylor, JL.; Orsmond, GI.; Esbensen, A.; Hong, JY. Adolescents and adults with autism spectrum disorders. In: Amaral, DG.; Dawson, G.; Geschwind, D., editors. Autism spectrum disorders. New York: Oxford University Press; 2011.

Seltzer MM, Krauss MW, Shattuck PT, Orsmond G, Swe A, Lord C. The symptoms of autism spectrum disorders in adolescence and adulthood. Journal of Autism and Developmental Disorders. 2003; 33(6):565-581. [PubMed: 14714927]

Shields J, Konold TR, Glutting JJ. Validity of the Wide Range Intelligence Test: differential effects across race/ethnicity, gender, and education level. Journal of Psychoeducational Assessment. 2004; 22(4):287.

Sparrow, S.; Balla, DA.; Cicchetti, DV. Vineland Adaptive Behavior Scales. Circle Pines, MN: American Guidence Service; 1984.

Sparrow, S.; Carter, A.; Cicchetti, DV. Vineland Screener: Overview, reliability, validity, administration, and scoring. New Haven: Yale University Child Study Center; 1993. 
Sutherland A, Crewther DP. Magnocellular visual evoked potential delay with high autism spectrum quotient yields a neural mechanism for altered perception. Brain. 2010; 133(Pt 7):20892097.10.1093/brain/awq122 [PubMed: 20513659]

Takagishi H, Takahashi T, Yamagishi T, Shinada M, Inukai K, Tanida S, et al. Salivary testosterone levels and autism-spectrum quotient in adults. Neuro Endocrinol Lett. 2010; 31(6):837-841. [PubMed: 21196912]

Taylor J, Seltzer M. Changes in the autism behavioral phenotype during the transition to adulthood. Journal of Autism and Developmental Disorders. 2010:1-16.10.1007/s10803-010-1005-Z [PubMed: 19633942]

Taylor JL, Seltzer MM. Employment and post-secondary educational activities for young adults with autism spectrum disorders during the transition to adulthood. Journal of Autism and Developmental Disorders. 2011; 41:566-574. [PubMed: 20640591]

Wakabayashi A, Baron-Cohen S, Wheelwright S. Are autistic traits an independent personality dimension? A study of the Autism-Spectrum Quotient (AQ) and the NEO-PI-R. Personality and Individual Differences. 2006; 41(5):873-883.

Wakabayashi A, Tojo Y, Baron-Cohen S, Wheelwright S. The Autism-Spectrum Quotient (AQ) Japanese version: Evidence from high-functioning clinical group and normal adults. Shinrigaku kenkyu: The Japanese journal of psychology. 2004; 75(1):78. [PubMed: 15724518]

Wheelwright S, Auyeung B, Allison C, Baron-Cohen S. Defining the broader, medium and narrow autism phenotype among parents using the autism spectrum quotient (AQ). Mol Autism. 2010; 1(1):10.10.1186/2040-2392-1-10 [PubMed: 20678260]

Whitehouse AJO, Barry JG, Bishop DVM. The broader language phenotype of autism: A comparison with specific language impairment. Journal of Child Psychology and Psychiatry. 2007; 48(8):822830. [PubMed: 17683454]

Woodbury-Smith MR, Robinson J, Wheelwright S, Baron-Cohen S. Screening adults for Asperger syndrome using the AQ: A preliminary study of its diagnostic validity in clinical practice. Journal of Autism and Developmental Disorders. 2005; 35(3):331-335. [PubMed: 16119474] 


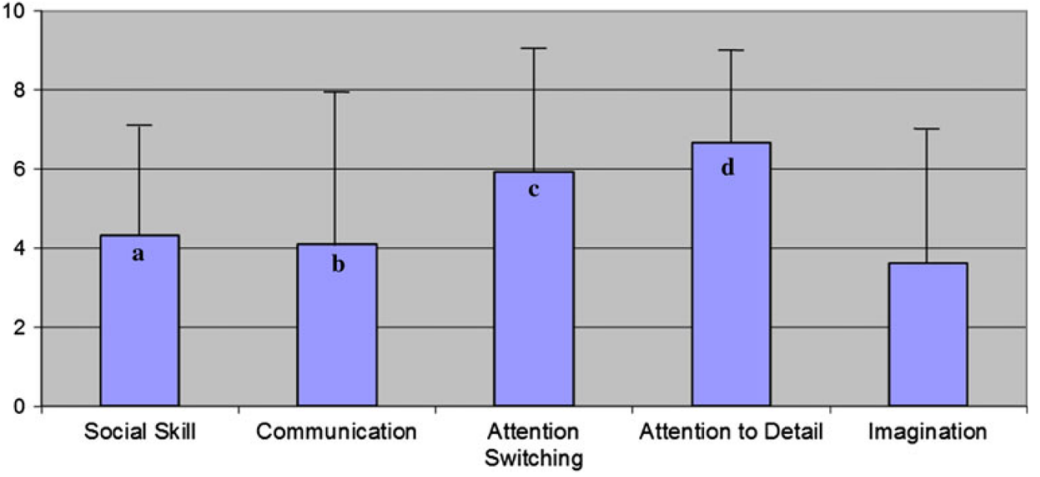

Fig. 1.

AQ subscale scores. $a$ Lower than Attention Switching: $t(64)=-4.94, p<.001$; Lower than Attention to Detail: $t(64)=-4.93, p<.001$. $b$ Lower than Attention Switching: $t(64)=$ $-6.22, p<.001$; Lower than Attention to Detail: $t(64)=-6.35, p<.001$. $c$ Higher than Imagination: $t(64)=7.76, p<.001$. $d$ Higher than Imagination: $t(64)=9.08, p<.001$ 


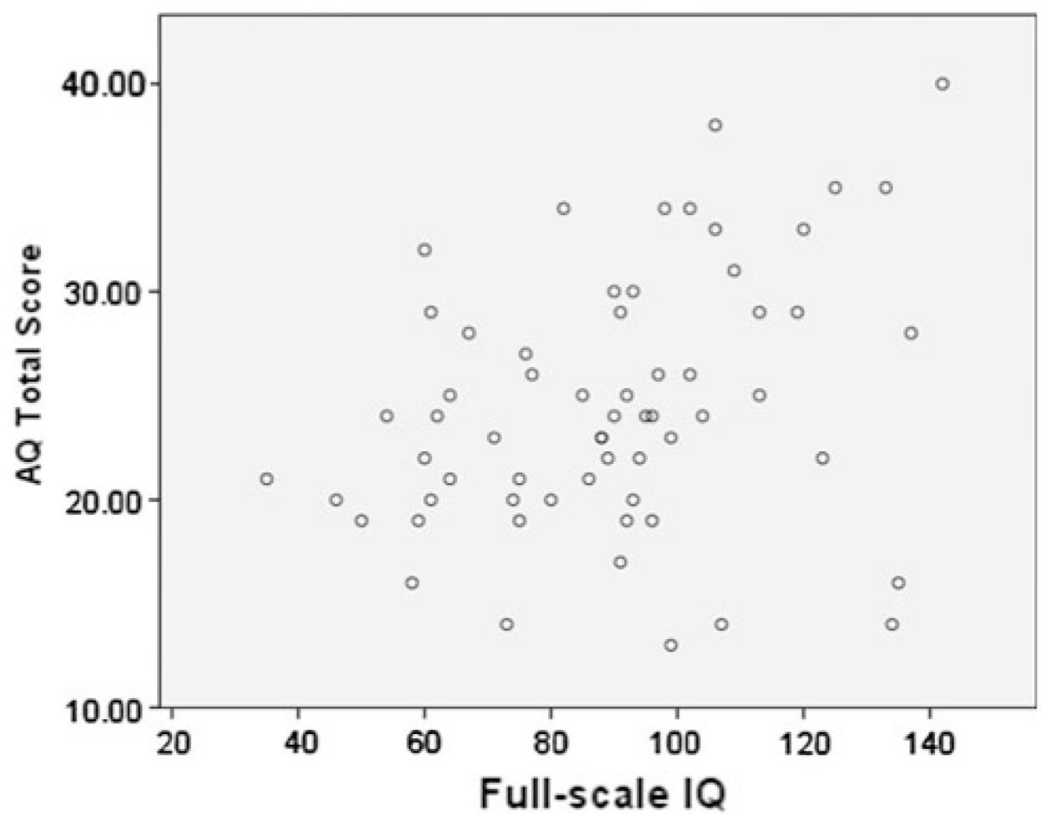

Fig. 2.

Correlation between AQ scores and Full-scale IQ 
Table 1

Participant demographics $(n=65)$

\begin{tabular}{ll}
\hline Males & $49(75 \%)$ \\
Females & $16(25 \%)$ \\
White & $62(95 \%)$ \\
Non-white & $3(5 \%)$ \\
Co-residing with parent & $44(68 \%)$ \\
Living outside of parental home & $21(32 \%)$ \\
Participant age (years) & $\mathrm{M}=24.97, \mathrm{SD}=8.22$ (range: $18.0-52.6)$ \\
Mother age (years) & $\mathrm{M}=54.73, \mathrm{SD}=9.01$ (range: $39.3-83.9)$ \\
Full-scale IQ (WRIT) & $\mathrm{M}=89.61, \mathrm{SD}=24.23$ (range: $35-142)$ \\
Vineland Screener composite & $\mathrm{M}=64.33, \mathrm{SD}=14.55$ (range: $35-100)$ \\
\hline
\end{tabular}


Table 2

\section{AQ item endorsement}

\begin{tabular}{|c|c|c|}
\hline Item & & $\%$ Score $=1$ \\
\hline & Social skill & \\
\hline 1 & I prefer to do things with others rather than on my own. & 26 \\
\hline 11 & I find social situations easy. & 49 \\
\hline 13 & I would rather go to a library than a party. & 42 \\
\hline 15 & I find myself drawn more strongly to people than to things. & 43 \\
\hline 22 & I find it hard to make new friends. & 74 \\
\hline 36 & I find it easy to work out what someone is thinking or feeling just by looking at their face. & 49 \\
\hline 44 & I enjoy social occasions. & 22 \\
\hline 45 & I find it difficult to work out people's intentions. & 74 \\
\hline 47 & I enjoy meeting new people. & 17 \\
\hline \multirow[t]{2}{*}{48} & I am a good diplomat. & 35 \\
\hline & Attention Switching & \\
\hline 2 & I prefer to do things the same way over and over again. & 68 \\
\hline 4 & I frequently get so strongly absorbed in one thing that I lose sight of other things. & 72 \\
\hline 10 & In a social group, I can easily keep track of several different people's conversations. & 43 \\
\hline 16 & I tend to have very strong interests, which I get upset about if I can't pursue. & 66 \\
\hline 25 & It (does not) upsets me if my daily routine is disturbed. & 72 \\
\hline 32 & I find it easy to do more than one thing at once. & 51 \\
\hline 34 & I enjoy doing things spontaneously. & 28 \\
\hline 37 & If there is an interruption, I can switch back to what I was doing very quickly. & 35 \\
\hline 43 & I like to plan any activities I participate in carefully. & 77 \\
\hline \multirow[t]{2}{*}{46} & New situations make me anxious. & 82 \\
\hline & Attention to Detail & \\
\hline 5 & I often notice small sounds when others do not. & 74 \\
\hline 6 & I usually notice car number plates or similar strings of information. & 77 \\
\hline 9 & I am fascinated by dates. & 62 \\
\hline 12 & I tend to notice details that others do not. & 74 \\
\hline 19 & I am fascinated by numbers. & 68 \\
\hline 23 & I notice patterns in things all the time. & 74 \\
\hline 28 & I usually concentrate more on the whole picture, rather than the small details. & 29 \\
\hline 29 & I am (not) very good at remembering phone numbers. & 69 \\
\hline 30 & I (don't) usually notice small changes in a situation, or a person's appearance. & 72 \\
\hline \multirow[t]{2}{*}{49} & I am (not very) good at remembering people's date of birth. & 34 \\
\hline & Communication & \\
\hline 7 & Other people frequently tell me that what I've said is impolite, even though I think it is polite. & 43 \\
\hline 17 & I enjoy social chit-chat. & 31 \\
\hline 18 & When I talk, it is (not) always easy for others to get a word in edgewise. & 63 \\
\hline 26 & I frequently find that I (don't) know how to keep a conversation going. & 31 \\
\hline 27 & I find it easy to "read between the lines" when someone is talking to me. & 49 \\
\hline
\end{tabular}

J Autism Dev Disord. Author manuscript; available in PMC 2012 November 01. 


\begin{tabular}{lll}
\hline Item & & \% Score = 1 \\
\hline 31 & I know how to tell if someone listening to me is getting bored. & 39 \\
33 & When I talk on the phone, I'm (not) sure when it's my turn to speak. & 20 \\
35 & I am often the last to understand the point of a joke. & 51 \\
38 & I am good at social chit-chat. & 71 \\
39 & People often tell me that I keep going on and on about the same thing. & \\
& Imagination & 14 \\
3 & If I try to imagine something, I find it very easy to create a picture in my mind. \\
8 & When I'm reading a story, I can easily imagine what the characters might look like. \\
14 & I find making up stories easy. & 22 \\
20 & When I'm reading a story, I find it difficult to work out the characters' intentions. \\
21 & I (don't) particularly enjoy reading fiction. & 54 \\
24 & I would rather go to the theatre than a museum. & 17 \\
40 & When I was young, I used to enjoy playing games involving pretending with other children. & 17 \\
41 & I like to collect information about categories of things (e.g. types of car etc.). & 75 \\
42 & I find it difficult to imagine what it would be like to be someone else. & 60 \\
50 & I find it very easy to play games with children that involve pretending. & 40 \\
\hline
\end{tabular}


Table 3

AQ total score correlations (Pearson's $r$ )

\begin{tabular}{lcccc}
\hline & Vineland social & ADI-R total & Age & Full-scale IQ \\
\hline AQ total score & -0.05 & 0.18 & -0.001 & 0.33 \\
Vineland social domain score & & $-0.66^{*}$ & -0.04 & 0.14 \\
ADI-R current overall total score & & 0.17 & -0.12 \\
Chronological age & & & -0.20 \\
\hline$*$ & & & \\
$p<.001$ & & &
\end{tabular}


Table 4

AQ total and subscale scores by IQ Group

\begin{tabular}{|c|c|c|c|}
\hline & Below average IQ $(n=23)$ & Average or above IQ $(n=39)$ & Group comparison \\
\hline Full-scale IQ & $\begin{array}{l}\mathrm{M}=64.52, \mathrm{SD}=11.57, \text { Range: } 35- \\
82\end{array}$ & $\begin{array}{l}M=104.41, S D=16.10, \text { Range: } 85- \\
142\end{array}$ & $t(60)=-10.39, p<.001 *$ \\
\hline AQ total score & $\mathrm{M}=22.78, \mathrm{SD}=4.86$, Range: $14-34$ & $\mathrm{M}=25.62, \mathrm{SD}=6.69$, Range: $13-40$ & $t(60)=-1.77, p=.08$ \\
\hline Social Skill & $\mathrm{M}=3.13, \mathrm{SD}=1.69$, Range: $1-7$ & $\mathrm{M}=4.90, \mathrm{SD}=2.76$, Range: $0-10$ & $t(60)=-2.77, p=.007^{*}$ \\
\hline Attention Switching & $\mathrm{M}=5.22, \mathrm{SD}=2.07$, Range: $1-9$ & $\mathrm{M}=6.26, \mathrm{SD}=2.01$, Range: $1-10$ & $t(60)=-1.95, p=.06$ \\
\hline Attention to Detail & $\mathrm{M}=6.78, \mathrm{SD}=1.54$, Range: $2-9$ & $\mathrm{M}=6.77, \mathrm{SD}=2.28$, Range: $0-10$ & $t(60)=0.03, p=.98$ \\
\hline Communication & $\mathrm{M}=3.57, \mathrm{SD}=1.65$, Range: $1-8$ & $\mathrm{M}=4.25, \mathrm{SD} S D=2.40$, Range: $0-10$ & $t(60)=-1.22, p=.23$ \\
\hline Imagination & $\mathrm{M}=4.09, \mathrm{SD}=1.70$, Range: $1-7$ & $\mathrm{M}=3.44, \mathrm{SD}=1.83$, Range: $0-8$ & $t(60)=1.39, p=.17$ \\
\hline $\begin{array}{l}\text { Met diagnostic cutoff of } \\
32\end{array}$ & $2(9 \%)$ & $8(21 \%)$ & $\chi^{2}(1, N=62)=1.49, p=.30$ \\
\hline Met screening cutoff of 26 & $6(26 \%)$ & $17(44 \%)$ & $\chi^{2}(1, N=62)=1.90, p=.19$ \\
\hline
\end{tabular}

